University of Wollongong

Research Online

Australian Institute for Innovative Materials -

Papers

Australian Institute for Innovative Materials

$1-1-2011$

Compact-designed supercapacitors using free-standing single-walled carbon nanotube films

Zhiqiang Niu

Chinese Academy of Science, Beijing

Weiya Zhou

Chinese Academy of Sciences, Beijing

Jun Chen

University of Wollongong, junc@uow.edu.au

Guoxing Feng

Chinese Academy of Sciences, Beijing

Hong Li

Chinese Academy of Sciences, Beijing

See next page for additional authors

Follow this and additional works at: https://ro.uow.edu.au/aiimpapers

Part of the Engineering Commons, and the Physical Sciences and Mathematics Commons

Research Online is the open access institutional repository for the University of Wollongong. For further information contact the UOW Library: research-pubs@uow.edu.au 


\title{
Compact-designed supercapacitors using free-standing single-walled carbon nanotube films
}

\author{
Abstract \\ We reported the realization of assembling compact-designed supercapacitors using large-scaled \\ freestanding and flexible single-walled carbon nanotube (SWCNT) films as both anode and cathode. A \\ prototype of the processing procedures was developed to obtain the uniform spreading of the SWCNT \\ films onto the separators serving as both electrodes and charge collectors without metallic current \\ collectors, leading to a simplified and lightweight architecture. The area of SWCNT film on a separator \\ can be scaled up and its thickness can be extended. High energy and power densities $\left(43.7 \mathrm{Wh} \mathrm{kg}^{-1}\right.$ and \\ $197.3 \mathrm{~kW} \mathrm{~kg}^{-1}$, respectively) were achieved from the prepared SWCNT film-based compact-designed \\ supercapacitors with small equivalent series resistance. The specific capacitance of this kind of \\ compact-designed SWCNT film supercapacitor is about $35 \mathrm{~F} \mathrm{~g}^{-1}$. These results clearly show the potential \\ application of free-standing SWCNT film in compact-designed supercapacitor with enhanced \\ performance and significantly improved energy and power densities.
}

\section{Keywords}

walled, carbon, single, standing, compact, free, films, supercapacitors, nanotube, designed

Disciplines

Engineering | Physical Sciences and Mathematics

\section{Publication Details}

Niu, Z, Zhou, W, Chen, J, Feng, G, Li, H, Ma, W, Li, J, Dong, H, Ren, Y, Zhao, D \& Xie, S (2011), Compactdesigned supercapacitors using free-standing single-walled carbon nanotube films, Energy and Environmental Science, 4(4), pp. 1440-1446.

\section{Authors}

Zhiqiang Niu, Weiya Zhou, Jun Chen, Guoxing Feng, Hong Li, Wenjun Ma, Jinzhu Li, Haibo Dong, Yan Ren, Duan Zhao, and Sishen Xie 


\title{
Energy \& Environmental Science
}

\section{Compact-designed supercapacitors using free-standing single-walled carbon nanotube films}

\author{
Zhiqiang Niu, ${ }^{a c}$ Weiya Zhou, ${ }^{* a}$ Jun Chen, ${ }^{b}$ Guoxing Feng, ${ }^{a c}$ Hong Li, ${ }^{a}$ Wenjun Ma, ${ }^{a c}$ Jinzhu Li, ${ }^{a c}$ \\ Haibo Dong, ${ }^{a c}$ Yan Ren, ${ }^{a c}$ Duan Zhao ${ }^{a c}$ and Sishen Xie ${ }^{* a}$
}

Received 14th July 2010, Accepted 10th January 2011

DOI: 10.1039/c0ee00261e

\begin{abstract}
We reported the realization of assembling compact-designed supercapacitors using large-scaled freestanding and flexible single-walled carbon nanotube (SWCNT) films as both anode and cathode. A prototype of the processing procedures was developed to obtain the uniform spreading of the SWCNT films onto the separators serving as both electrodes and charge collectors without metallic current collectors, leading to a simplified and lightweight architecture. The area of SWCNT film on a separator can be scaled up and its thickness can be extended. High energy and power densities $\left(43.7 \mathrm{Wh} \mathrm{kg}^{-1}\right.$ and $197.3 \mathrm{~kW} \mathrm{~kg}^{-1}$, respectively) were achieved from the prepared SWCNT film-based compact-designed supercapacitors with small equivalent series resistance. The specific capacitance of this kind of compact-designed SWCNT film supercapacitor is about $35 \mathrm{~F} \mathrm{~g}^{-1}$. These results clearly show the potential application of free-standing SWCNT film in compact-designed supercapacitor with enhanced performance and significantly improved energy and power densities.
\end{abstract}

\section{Introduction}

As energy storage devices, supercapacitors, also known as electrochemical capacitors, have attracted much attention because of their high power and energy densities and long cycle life. ${ }^{1-3}$

a Beijing National Laboratory for Condensed Matter Physics, Institute of Physics, Chinese Academy of Sciences, Beijing, 100190, China. E-mail. wyzhou@aphy.iphy.ac.cn; Fax: +86-10-82640215; Tel: +86-1082649381; ssxie@aphy.iphy.ac.cn

${ }^{b} A R C$ Centre of Excellence for Electromaterials Science, Intelligent Polymer Research Institute, University of Wollongong, Northfields Avenue, Wollongong, NSW, 2522, Australia

${ }^{c}$ Graduate School of the Chinese Academy of Sciences, Beijing, 100039, China
Supercapacitors could be applied to many fields such as electric vehicles, pulse power applications and portable devices. ${ }^{4}$ These applications require that the supercapacitors have not only high performance, but also small size and light weight. The supercapacitors can be of various designs including, but not limited to, stacked and rolled (also known as spiral-wound) configurations. Regardless of stacked or rolled configurations, large-area electrode materials can offer large capacitance. The stacked design determines that stacked supercapacitors with large-area electrode materials will be bulky, which will limit the use of the stacked supercapacitors in the applications that are limited in space. By contrast with the stacked design, with regard to rolled design, large-area electrode materials can be used and large capacitance will be achieved without becoming too heavy or

\section{Broader context}

As energy storage devices, supercapacitors can be applied to many fields owing to their high power and energy densities and long cycle life. Currently, activated carbon (AC) materials are the typical electrode material of supercapacitors. However, because the equivalent series resistance of supercapacitors based on AC materials is high, the energy and power densities are low for the commercially available supercapacitors based on AC electrodes. The high conductivity, low mass density and large specific surface area of single-walled carbon nanotubes (SWCNTs) make them attractive as high performance electrode material of supercapacitors. To meet the demand for high-capacitance and small-size of efficient power devices, SWCNT film-based compact supercapacitors provide a possibility of ideal compromise. Most of the reported supercapacitors based on SWCNT film electrodes are stacked design, because the SWCNT films with substrates used as supercapacitor electrodes are not flexible and the area of SWCNT fims is difficult to scale up. In this work, we realized the assembly compact-designed supercapacitors using large-scaled free-standing and flexible SWCNT films as both anode and cathode. The area and thickness of SWCNT film electrodes can be scaled up. High energy and power densities were achieved. 
bulky. Therefore, to meet the high-capacitance and small-size characteristics for efficient power devices, rolled design seems to be the possibility of an ideal compromise.

The performance characteristics of energy storage devices are fundamentally determined by the structural and electrochemical properties of electrode materials. Rolled design of supercapacitors requires that electrode materials have to be flexible. Currently, activated carbon (AC) materials are the typical electrode material of spiral-wound supercapacitor. ${ }^{5,6}$ But the preparation of AC material-based electrodes is complicated and the equivalent series resistance (ESR) of supercapacitors based on AC materials is high due to the low conductivity and electrolyte accessibility of the AC material-based electrodes. ${ }^{6,7}$ As a result, the commercially available electrochemical capacitors based on AC electrodes possess a low energy density (1-10 $\mathrm{Wh} \mathrm{kg}^{-1}$ ) and a low power density $\left(2-10 \mathrm{~kW} \mathrm{~kg}^{-1}\right) .{ }^{\mathbf{4}, 8}$ In order to apply supercapacitors to various practical devices such as electric vehicles, it is necessary to improve the power density and energy density of supercapacitors. Single-walled carbon nanotubes (SWCNTs) are the attractive candidate for high performance electrode material of supercapacitors, because SWCNTs have high conductivity, low mass density, large specific surface area and high mechanical strength. ${ }^{9-19}$ SWCNT-based electrodes have been achieved by mixing SWCNTs with conductive binders and coating collector electrodes with this composite. ${ }^{20}$ The addition of binders degraded the properties of SWCNT electrodes, resulting in undesirable performance of the supercapacitors. For example, the binders will block some pores of porous SWCNT film and increase the internal resistance of the electrodes, leading to lower performance of the supercapacitors. Besides, postdeposited SWCNT film on the substrates can be used as electrode materials for the supercapacitor and excellent results have been reported. ${ }^{21-24}$ However, these SWCNT films used as supercapacitor electrodes have to be deposited onto the substrates and the resulting SWCNT films with substrates are not flexible and tough enough. SWCNT arrays were also used as the electrodes of supercapacitors and ultrahigh energy density and power density were achieved. ${ }^{19,25}$ But the area of SWCNT arrays is limited and difficult to scale up, which could be the reason that the reported supercapacitors based on SWCNT array electrodes in general are stacked design. The major challenge in realization of rolled design for SWCNT film supercapacitors is the fabrication of large-area flexible conducting SWCNT film electrodes.

Here, we propose a novel prototype of the processing procedures to obtain the uniform spreading of the SWCNT films onto the separators. This method can scale up the area of SWCNT film on a separator and extend its thickness. The compact design of supercapacitors was realized using flexible SWCNT films on the separators as both electrodes and charge collectors without metallic current collectors. Comparing with stacked electrodes, the area of electrodes in rolled design can be scaled up. Large capacitance will be achieved without becoming too heavy or bulky. Excellent performance such as high energy and power densities was achieved for the prepared compactdesigned SWCNT film supercapacitors with small ESR. These clearly show the potential application of free-standing SWCNT film in a compact-designed supercapacitor with enhanced performance and significantly improved energy and power densities.

\section{Experimental details}

\section{Fabrication process of supercapacitor in compact design}

The SWCNT films used as both electrodes and charge collectors were directly prepared by the floating chemical vapor deposition method. ${ }^{26}$ For preparing electrodes of supercapacitors in rolled design, the SWCNT films and the separators (Celgard 2325) were cut into a certain size to match the demand of the designed supercapacitor electrodes, respectively (shown in Fig. 1a). Then, many tailored SWCNT films and a tailored separator were dipped into ethanol as shown in Fig. 1b. In ethanol, these SWCNT films can be easily spread out onto the separator end to end or/ and layer by layer. When the SWCNT films were spread out onto the separators end to end, the ends of neighboring SWCNT films would be overlapped, as shown in Fig. 1c. This could effectively avoid the presence of uncovered blank regions and ensure good contact between the SWCNT films. It was found that the small overlapping regions less than $1 \mathrm{~mm}$ usually, relative to the size of the films, would have little effect on the uniform of the SWCNT films on the separators. The quantity and configuration of the SWCNT films on the separator can be adjusted, according to the practical demand. After uniformly spreading out SWCNT films onto the separator, the ethanol was evaporated and the SWCNT films can adhere firmly to the separator, as shown in Fig. 1c. Then, two pieces of separator coated with SWCNT films were stacked together and rolled up, followed with the assembly of the compact-designed supercapacitor by filling with $1 \mathrm{M} \mathrm{LiClO}_{4}$ in a mixture of ethylene carbonate (EC), diethyl carbonate (DEC), and dimethylene carbonate (DMC) in a volume ratio of $\mathrm{EC} / \mathrm{DEC} / \mathrm{DMC}=1: 1: 1$ as the electrolyte, as shown in Fig. 1d and $1 \mathrm{e}$.

\section{Characterization}

Cyclic voltammetry (CV) of the SWCNT film supercapacitors was performed by $\mathrm{CHI} 660 \mathrm{C}$ instrument (CHI Instruments). The galvanostatic charge-discharge of the SWCNT film supercapacitors at a high operation voltage range ( 0 to $3.0 \mathrm{~V})$ was carried out on a supercapacitor test system (BT2000 Arbin). The morphology and microstructures of the SWCNT films were characterized by scanning electron microscopy (SEM, Hitachi S5200) and transmission electron microscopy (TEM, JEOL JEM2010).

\section{Results and discussion}

The optical image of directly grown SWCNT film (Fig. 2a) shows a large-scale (over $50 \mathrm{~cm}^{2}$ ), free-standing and homogeneous film with a thickness of about $400 \mathrm{~nm}$, which provides us with the opportunity to tailor this kind of SWCNT film into any desired shape as electrode material to match the device requirement. The free-standing SWCNT film also exhibits high tensile strength of $250 \mathrm{MPa},{ }^{26}$ which is 20 times higher than a typical bulky paper. ${ }^{27}$ Good strength and toughness ensure that the SWCNT film can be handled easily and can maintain its structure and integrity in the rolling process for electrode fabrication. Besides, the directly prepared SWCNT film is highly conducting with a very low sheet resistance in the range of 5-50 ohm square ${ }^{-1}$ depending on the film thickness from 500 to $100 \mathrm{~nm},{ }^{26}$ which can effectively 


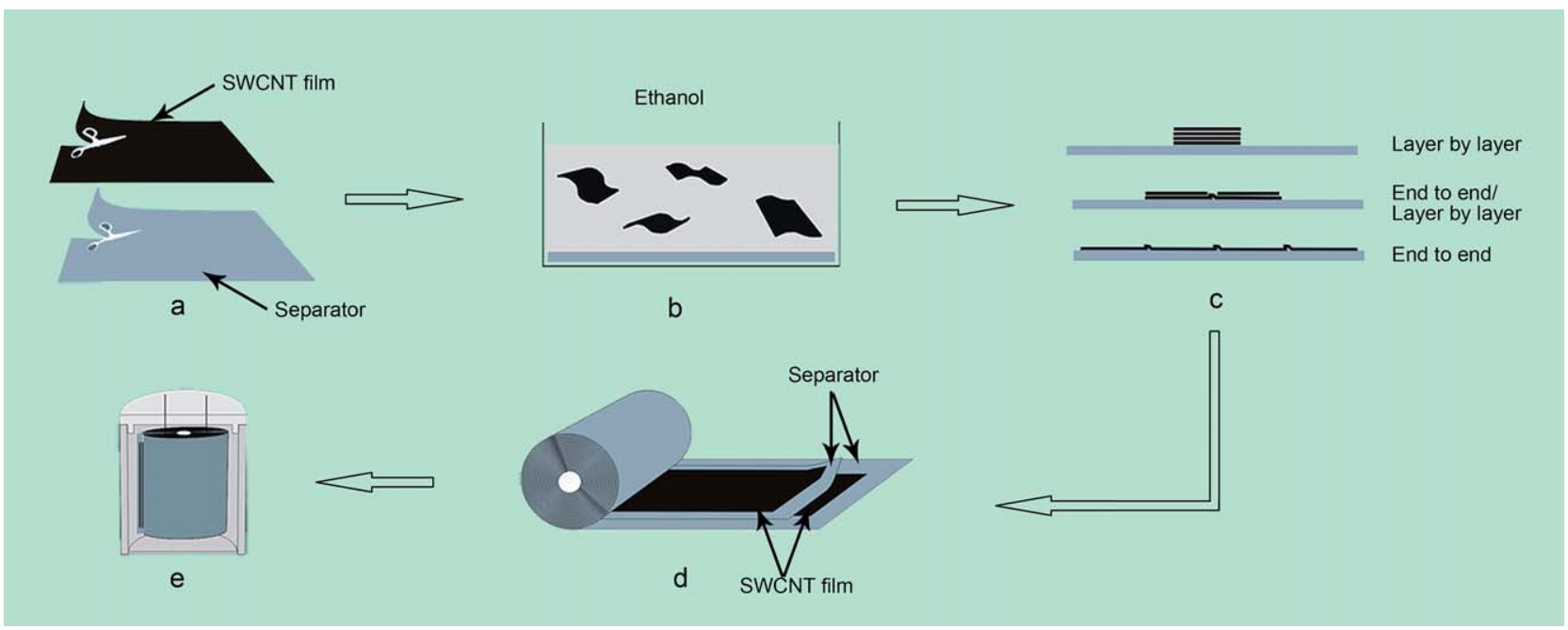

Fig. 1 Schematic diagram of assembling compact-designed supercapacitor using free-standing flexible SWCNT films.
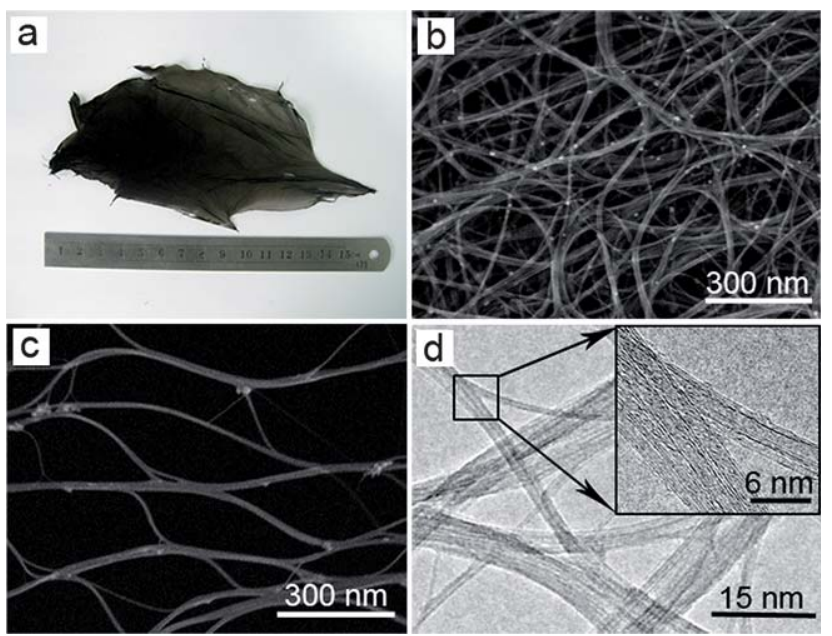

Fig. 2 (a) Optical image and (b) SEM image of the directly grown SWCNT film. (c) SEM image of a single layer of bundles peeled off from thick SWCNT film. (d) TEM image of the directly grown SWCNT film.

decrease the ESR of supercapacitors. Due to these unique excellent properties of the directly grown free-standing SWCNT film, such as flexibility, high electrical conductivity and largescale size, it has the potential to be an electrode material for the compact-designed supercapacitors.

SEM image of the free-standing SWCNT film (Fig. 2b) shows a nanoporous architecture with less visible impurity, which would be of great significance for its application in electrochemical devices. Fig. 2c displays a typical SEM image of SWCNT bundles peeled off from the thick film, which clearly illustrates that the SWCNT bundles in the film firmly connect with each other and form a continuous 2-D reticulate structure. This unique architecture plays a key role in achieving higher strength and higher conductivity of the directly grown SWCNT films than other post-deposited CNT films such as Bucky paper, which have a short and random-oriented structure. The TEM image of SWCNTs in the film (Fig. 2d) shows that the sidewall of SWCNTs is almost amorphous carbon free. It might be useful to improve the specific capacitance of the SWCNT film electrodes. ${ }^{19,28}$ Their high surface energy and extremely high surfaceto-volume ratio could lead to the strong self-adhesion of the directly grown SWCNT films. ${ }^{29}$

Although the large-area directly grown SWCNT films can be easily tailored into any desired shapes and sizes to produce and optimize various designs of supercapacitors, it is difficult to achieve uniform SWCNT film electrodes by directly spreading out SWCNT films onto separators due to the strong self-adhesion of the directly grown SWCNT films. As previously reported, ${ }^{29}$ the as-prepared SWCNT films treated by ethanol no longer have strong self-adhesion due to the reduced surface energy. Thus, in ethanol, the SWCNT films can be easily spread out onto the separator end to end or/and layer by layer according to the demand of the designed supercapacitor electrodes, as shown in Fig. $1 \mathrm{~b}$ and 1c. The quantity and configuration of the SWCNT films on the separator can be adjusted, according to the practical demand. After uniformly spreading out SWCNT films onto the separator, the ethanol was evaporated and the SWCNT films can adhere to the separator and ends of neighboring SWCNT films would be overlapped closely and firmly due to the partial recovery of self-adhesion property of the film. Then, two pieces of separator coated with SWCNT films were stacked together and rolled up, followed by the assembly of the compact-designed supercapacitor, as shown in Fig. 1d and 1e. It is worth noting that the shape and size of external shell of the resulting supercapacitor can be adjusted to various designs according to the practical applications.

The above demonstrated compact and rolled design of supercapacitor using free-standing SWCNT films as the electrode materials has some advantages over other traditional materials. First, the large-area flexible directly grown SWCNT film can be easily tailored into any desired shape and size, which would be of great significance for the design of various supercapacitors. Second, as we know, ethanol is easily evaporated so that it leaves the SWCNT film free of contamination after processing, which is very important for SWCNT films used as electrode materials in electrochemical devices. And in ethanol, the SWCNT film is no longer self-adhesive and can be easily 
manipulated because ethanol molecules were absorbed on the surface of SWCNTs and lowered the surface energy of the film. Therefore, the SWCNT films can be spread out onto the separators uniformly to match the demand of the designed supercapacitor electrodes. After the evaporation of ethanol, the self-adhesion property of SWCNT film will be partially restored. So the ends of neighboring SWCNT films would be overlapped closely and the contact resistance between neighboring SWCNT films is small, which is beneficial for the power density of supercapacitors. Third, not only can the area of SWCNT films on the separator be easily scaled up, but also the thickness of SWCNT films can be controlled by a layer-by-layer process. This offers the great opportunity to achieve a single supercapacitor with high enough capacitance, power density and energy density to match the requirements for practical applications.

From the optical image of a separator coated with SWCNT films (Fig. 3a), it is revealed that the SWCNT films had been

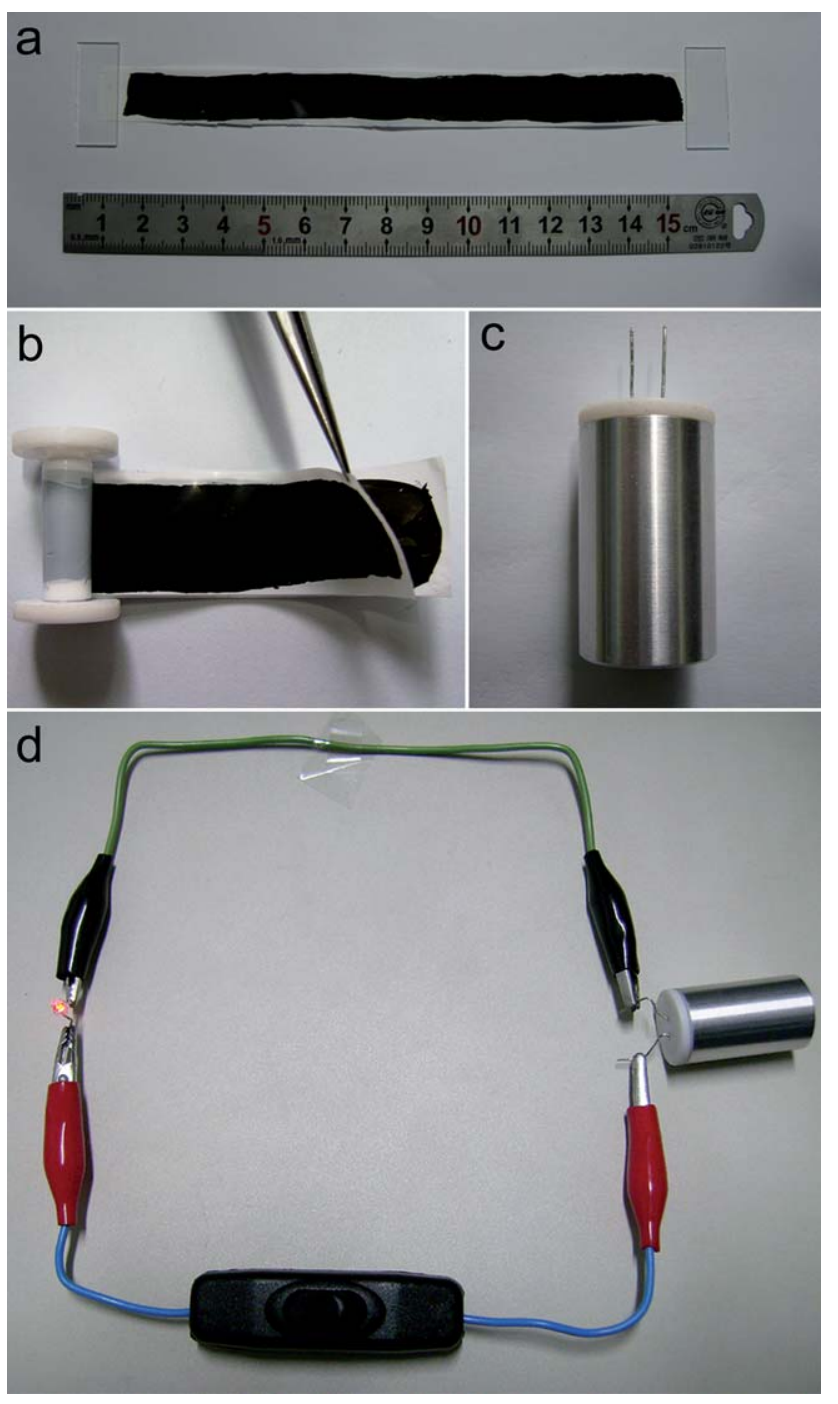

Fig. 3 Optical image of (a) the SWCNT films spread out on a separator, (b) rolled design of the separator with SWNCT films and (c) the resulting compact-designed supercapacitor. (d) Optical image of the resulting compact-designed supercapacitor used to glow a red light-emitting diode (LED). flattened onto the separator. After the evaporation of ethanol and drying in the air, the SWCNT films could not be peeled off from the separator any more, indicating good adhesion between SWCNT films and separator. Compared to AC materials and post-deposited SWCNT films, these directly grown SWCNT films have a significantly higher conductivity due to their unique continuous reticulate architecture, which makes it possible for them to be used as current collectors without extra metallic current collectors normally used in a traditional supercapacitor. Therefore, in our case, the SWCNT films were directly used as both electrodes and charge collectors, which not only eliminated the metal/SWCNT film interface, namely charge collector/electrode interface, but also simplified the surpercapacitor design with less weight. In contrast, extra current collectors (metallic foils or films) for both anode and cathode were required in the supercapacitor devices using AC materials or other post-deposited SWCNT films as electrode materials due to their poor conductivity. ${ }^{24,30}$ Fig. $3 \mathrm{~b}$ shows the optical image of the fabrication procedure on how to roll up two pieces of SWCNT film/ separator. The resulting compact-designed supercapacitor was illustrated in Fig. 3c. And this kind of SWCNT film-based compact-designed supercapacitor was powerful enough to light up a light-emitting diode (Fig. 3d) when fully charged. SWCNT films have a high electrochemical stability due to slow kinetics of carbon oxidation in comparison with AC materials. Together with the high conductivity and excellent mechanical strength, the free-standing SWCNT films have the potential to be the desirable replacement of AC materials in electrochemical energy devices, including the compact-designed supercapacitor with enhanced device performance.

The electrochemical characterization of the above compactdesigned supercapacitor was investigated by $\mathrm{CV}$ at different scan rates (Fig. 4a), showing a typical rectangular shape. This indicates that the SWCNT film has excellent capacitance behavior with very rapid current response on voltage reversal with low $\mathrm{ESR},{ }^{31}$ which is consistent with the results obtained from other stacked SWCNT-based supercapacitors. ${ }^{23}$ It is noteworthy that a significant deviation at higher potentials was observed in the $\mathrm{CV}$ curves, indicating the presence of pseudo-capacitance. However, in this work, we only used the directly grown SWCNT films without any further treatment as electrode materials for the fabrication of supercapactors. Therefore the pseudo-capacitance could not be from the SWCNTs and might be attributed to the remaining Fe catalyst particle in SWCNTs or the distribution of SWCNTs. ${ }^{12}$ It was reported that metallic nanoparticles could act as reversible redox sites. ${ }^{32}$ The featureless and rectangular-shaped CV curves suggest that the directly grown SWCNT films are an excellent candidate for electrochemical double-layer capacitors.

The specific capacitance $C_{\text {spe }}$ was estimated from CV curves using the equation ${ }^{31,33}$

$$
C_{\text {spe }}=I / s m
$$

where $I$ is the corresponding current of the voltage applied (at 1.5 $\mathrm{V}), s$ is the scan rate and $m$ is the total mass of the SWCNT films on both electrodes.

The calculated specific capacitance of the SWCNT film-based supercapacitor is about $34 \mathrm{~F} \mathrm{~g}^{-1}$ at a scan rate of $20 \mathrm{mV} \mathrm{s}^{-1}$. 

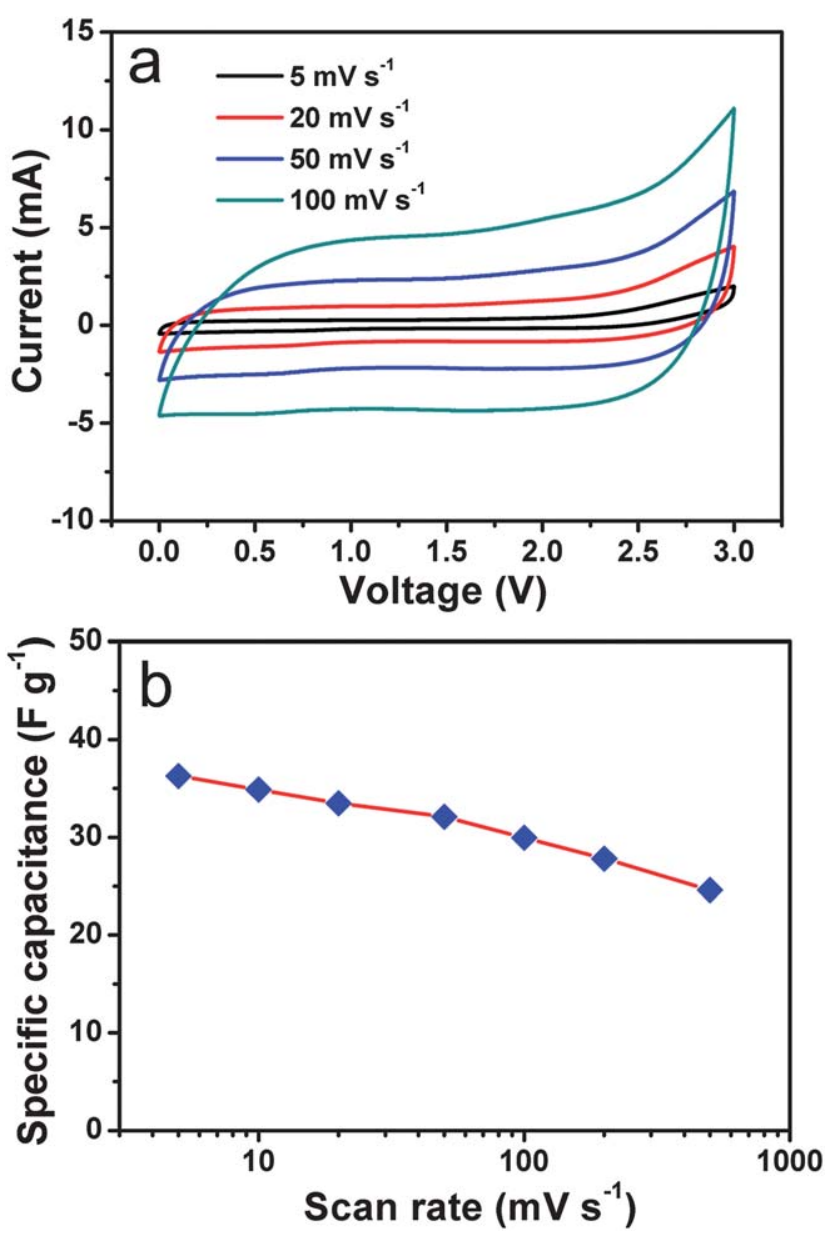

Fig. 4 (a) Typical CVs of a representative compact-designed SWCNT film supercapacitor at different scan rates. (b) The specific capacitance of the SWCNT film supercapacitor as a function of the scan rate.

Fig. 4b shows the specific capacitance of the SWCNT film supercapacitor as a function of the scan rate. The specific capacitance decreases continually with the increase of scan rate due to progressively less efficient infiltration of ions into the porous films at higher scan rates, a typical behavior of CNT film electrode. ${ }^{24}$ At slower scan rates, the diffusion of ions from the electrolyte can gain access to almost all available pores of the film electrodes and the penetration of electrolytic ions into the pores will be a lot deeper, leading to a high specific capacitance. However, when the scan rate is increased, the efficient infiltration of ions into the porous films is progressively lower, and as a result, there is a reduction in capacitance. ${ }^{31}$

The typical charge/discharge cycle of the compact-designed supercapacitor at $0.75 \mathrm{~A} \mathrm{~g}^{-1}$ in a high operation voltage range ( 0 to $3.0 \mathrm{~V}$ ) is shown in Fig. 5. It is known that each branch of the complete charge/discharge curve starts with a voltage drop (IR drop) because of internal resistance. However, almost no obvious IR drop was observed in Fig. 5, indicating a very low ESR for the SWCNT film-based supercapacitor. In addition, Fig. 5 also shows that the charge/discharge curves are perfectly linear, which demonstrates the capacitive behavior of the device with less Faradic reaction. ${ }^{31}$ The coulombic efficiency of the supercapacitor, $\eta$, was estimated as ${ }^{32}$

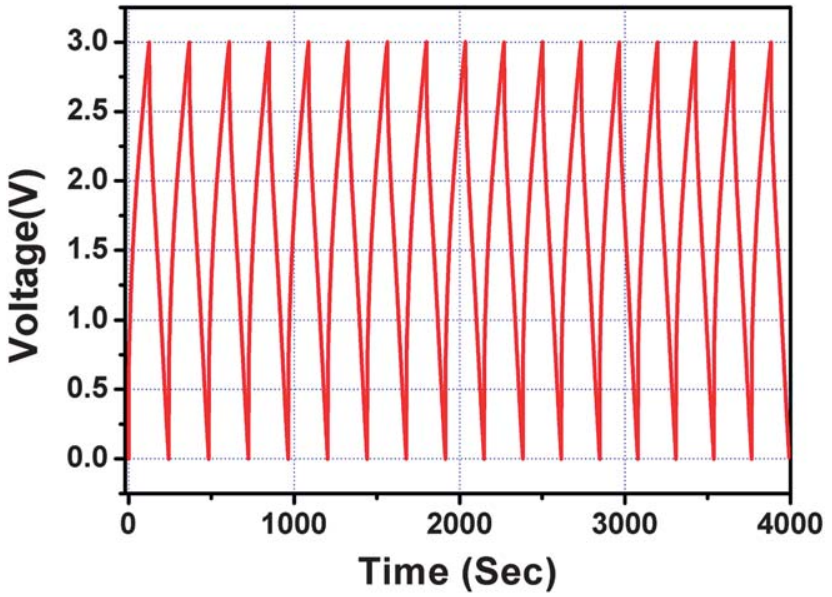

Fig. 5 Typical galvanostatic charge/discharge curves of the compactdesigned SWCNT film supercapacitor.

$$
\eta=\Delta t_{\mathrm{d}} / \Delta t_{\mathrm{c}} \times 100 \%
$$

where $\Delta t_{\mathrm{d}}$ and $\Delta t_{\mathrm{c}}$ represent the discharge and charge time, respectively. The coulombic efficiency nearly reached $95 \%$, implying good charge/discharge reversibility for the supercapacitor.

The specific capacitances $C_{\text {spe }}$ of supercapacitor devices were also calculated from constant current charge/discharge curves using the equation below ${ }^{31,34}$

$$
C_{\text {spe }}=\frac{I}{(\mathrm{~d} V / \mathrm{d} t) m}
$$

where $I$ is the discharge current, $\mathrm{d} V / \mathrm{d} t$ represents the slope of the discharge curve and $m$ is the total mass of the SWCNT film on both electrodes.

The specific capacitance of the resulting compact-designed SWCNT film supercapacitor is about $35 \mathrm{~F} \mathrm{~g}^{-1}$ for a two-electrode cell and corresponds to $140 \mathrm{~F} \mathrm{~g}^{-1}$ for a three-electrode cell, which is higher than those of a post-deposited CNT film or CNT array supercapacitors. ${ }^{23,31,35-37}$ This may be attributed to the high surface to volume ratio of SWCNTs. This strongly agrees with the results obtained from the previous $\mathrm{CV}$ measurements. In addition, the energy density $\left(W=C_{\text {spe }} V^{2} / 2\right.$, where $\mathrm{V}$ is the voltage applied) is $43.7 \mathrm{Wh} \mathrm{kg}^{-1}$ and the maximum power density $\left(P_{\max }=V^{2} / 4 R m\right.$, where $R$ is the internal resistance, which can be revealed in the Nyquist plots in Fig. 6a, $m$ is the total mass of the SWCNT films on both electrodes) of the resulting compactdesigned SWCNT film supercapacitor, is $197.3 \mathrm{~kW} \mathrm{~kg}^{-1} \cdot{ }^{23,25}$ The electrochemical performances of compact-designed SWCNT film supercapacitors were summarized in Table 1. A comparison of the supercapacitor performance between our SWCNT film-based compact-designed supercapacitor and other various CNT-based supercapacitors reveals that the power density of the resulting compact-designed supercapacitor is comparable to that of SWCNT array based supercapacitor recently reported by IzadiNajafabadi et al., ${ }^{19}$ but substantially higher than that of other CNT-based supercapacitors fabricated using different methods. The high conductivity of our SWCNT film electrodes and the optimized architecture of supercapacitors result in a low ESR 


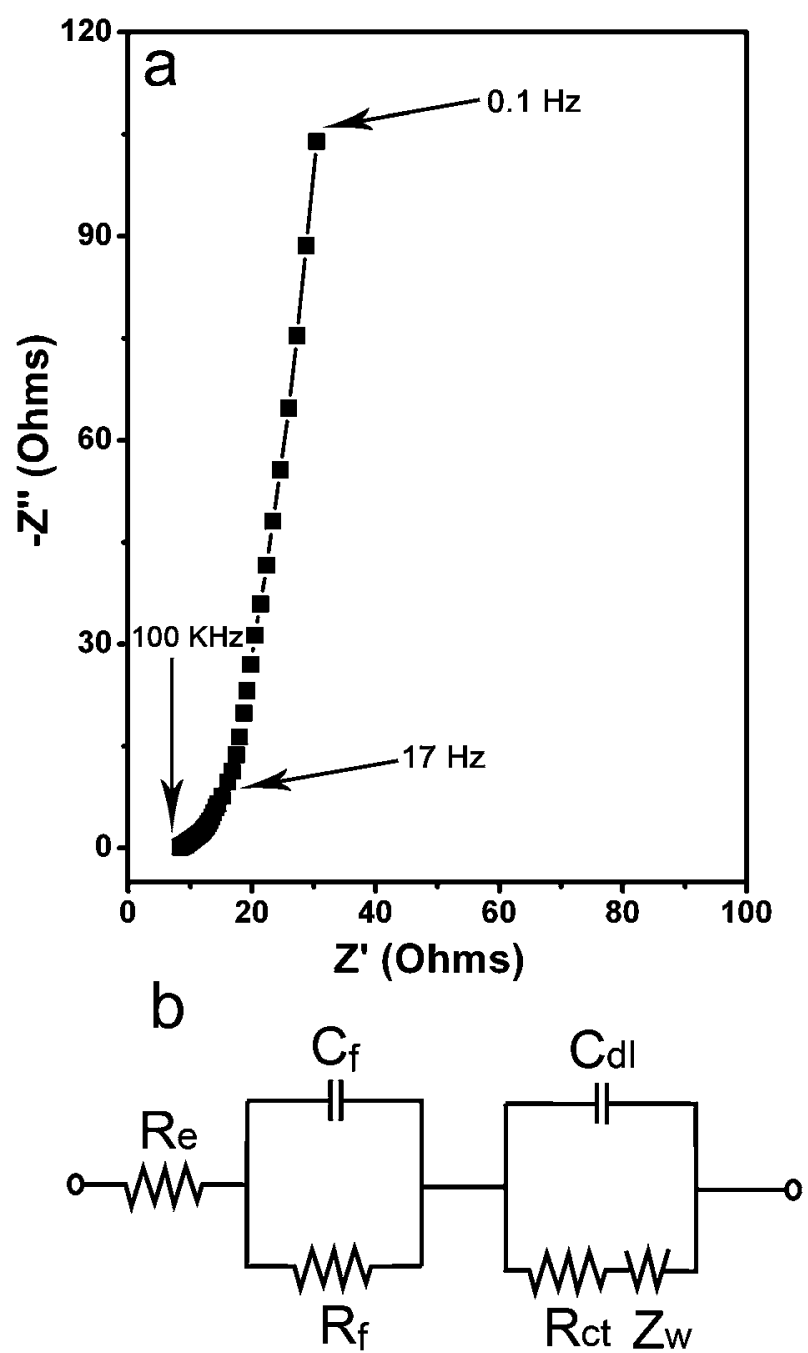

Fig. 6 (a) The Nyquist impedance plots of the compact-designed SWCNT film supercapacitor with frequency ranging from $100 \mathrm{kHz}$ to 0.1 Hz. (b) Equivalent circuit for the impedance spectra of directly grown SWCNT film electrodes.

and therefore a high power density of the SWCNT film-based supercapacitor in compact design.

Further electrochemical investigation was also carried out to understand in depth the device performance of SWCNT film supercapacitor in compact design. The impedance spectrum of the compact-designed supercapacitor is shown as Nyquist plots in Fig. 6a. At high frequencies, with the decrease of the frequency, Nyquist plot exhibits a "Warburg like" behaviour, which illustrates the expected shape for double layer charging of porous electrodes, consisting of a high frequency region with a slope of about $45^{\circ}$ and a low frequency region in which an capacitive behavior is approached. ${ }^{22,38}$ In Fig. 6a, it can be seen that the knee frequency is about $17 \mathrm{~Hz}$, indicating the availability of the rolled design of SWCNT film supercapacitor. It is noticeable that, at low frequencies, the Nyquist plot corresponding to the capacitive response of the SWCNT film electrodes is an almost vertical line, but not a rigorous vertical line. This may be ascribed to the different distribution of pore size of SWCNT film. ${ }^{30}$ The impedance plots can be explained using the equivalent circuit shown in Fig. $6 \mathrm{~b},{ }^{24,39}$ where $R_{\mathrm{e}}$ is the electrolyte resistance, $C_{\mathrm{f}}$ and $R_{\mathrm{f}}$ are the capacitance and resistance of SWCNT film electrodes, and $C_{\mathrm{d} 1}$ and $R_{\mathrm{ct}}$ are the double-layer capacitance and chargetransfer resistance, respectively; $Z_{\mathrm{w}}$ is the Warburg impedance related to the ionic diffusion in the SWCNT films. In Fig. 6a, no distinct semi-circle was observed. It is well known that the semicircle is related to the presence of an interface between the SWCNTs and the current collector and the electrical charge transfer in the electrode material due to the Faradic process. ${ }^{22,31}$ In our case, SWCNT films were directly used as both the electrodes and the charge collectors, and no other current collector was used. No distinct semi-circle indicates a very small charge transfer resistance $\left(R_{\mathrm{ct}}\right)$ at the SWCNT electrode/electrolyte interface, ${ }^{24}$ because the low thickness of SWCNT film favors the ion transfer kinetics of the SWCNT films. Previous SWCNT film electrodes prepared by other methods in general have micron scale thickness, but the thickness of the directly grown SWCNT film, which is applicable to rolled design of SWCNT film supercapacitors, can be extended up to $100 \mathrm{~nm}$. Regarding the nanoscale thickness of SWCNT film electrodes, the electrolyte could permeate into its pores and access the surface of interior SWCNTs easily, so that smaller charge transfer resistance was achieved. From Fig. 6a, we can estimate that the ESR for this particular device is about $200 \mathrm{~m} \Omega \mathrm{cm}^{-2}$, which is significantly lower than the reported values for other CNT film-based supercapcitors, as shown in Table 1. It suggests that directly grown SWCNT films could be a promising electrode material without any additional need of metallic collector for the supercapacitor with enhanced capacitive performance in rolled design.

\section{Conclusion}

A novel fabrication process has been developed to assemble the supercapacitor in rolled design using the directly grown SWCNT films as both the electrode and current collector, simplifying the architecture and reducing the weight of supercapacitors. An

Table 1 Comparison of the performance between the resulting compact-designed supercapacitors and the other various CNT-based supercapacitors

\begin{tabular}{|c|c|c|c|c|c|}
\hline Electrode type & Specific capacitance $\left(\mathrm{F} \mathrm{g}^{-1}\right)$ & Energy density $\left(\mathrm{Wh} \mathrm{kg}^{-1}\right)$ & Power density $\left(\mathrm{kW} \mathrm{kg}^{-1}\right)$ & $\operatorname{ESR}\left(\mathrm{m} \Omega \mathrm{cm}^{-2}\right)$ & Reference \\
\hline SWCNT film ${ }^{b, d}$ & 20 & 6 & 70 & $400-500$ & Ref. 12,36 \\
\hline MWCNT film ${ }^{b, d}$ & 21 & - & 20 & 1200 & Ref. 35 \\
\hline MWCNT array ${ }^{a, d}$ & $11-22$ & $2.3-5.4$ & $19.6-35.4$ & $>200$ & Ref. 31 \\
\hline
\end{tabular}


innovative strategy was used to uniformly spread out the SWCNT films onto the separator in controlled large-scale layered architectures, resolving the limitation in size and thickness of SWCNT film for the preparation of supercapacitors with high capacitance. The specific capacitance of the prepared compact-designed supercapacitors is about $35 \mathrm{~F} \mathrm{~g}^{-1}$. Significantly, due to the small ESR, high energy density (about 43.7 Wh $\mathrm{kg}^{-1}$ ) and power density (about $197.3 \mathrm{~kW} \mathrm{~kg}^{-1}$ ) were achieved for the SWCNT film-based compact-designed supercapacitors. The high electrical conductivity and excellent mechanical strength, in combination with enhanced electrochemical performance, suggest the directly grown free-standing SWCNT films have the significant potential to be promising electrode materials for small-size and lightweight energy storage devices. Compact design SWCNT film supercapacitors bring in new opportunities for advanced applications in energy storage devices with high performance and lightweight architecture.

\section{Acknowledgements}

This work is supported by the National Natural Science Foundation of China, the National Basic Research Program of China (Grant No. 2005CB623602), Beijing Municipal Education Commission (Grant No. YB20108000101) and the Key Item of Knowledge Innovation Project of the Chinese Academy of Sciences (KJCX2-YW-M01).

\section{Notes and references}

1 P. Simon and Y. Gogotsi, Nat. Mater., 2008, 7, 845-854.

2 L. L. Zhang and X. S. Zhao, Chem. Soc. Rev., 2009, 38, 2520-2531.

3 C. Liu, F. Li, L. P. Ma and H. M. Cheng, Adv. Mater., 2010, 22, E28E62.

4 Y. Zhang, H. Feng, X. B. Wu, L. Z. Wang, A. Q. Zhang, T. C. Xia, H. C. Dong, X. F. Li and L. S. Zhang, Int. J. Hydrogen Energy, 2009, 34, 4889-4899.

5 M. G. Deng, B. C. Yang and Y. D. HU, J. Funct. Mater., 2005, 36, 11821184.

6 A. Yoshida, S. Nonaka, I. Aoki and A. Nishino, J. Power Sources, 1996, 60, 213-218.

7 C. T. Hsieh and H. Teng, Carbon, 2002, 40, 667-674.

8 E. Frackowiak and F. Beguin, Carbon, 2001, 39, 937-950.

9 K. H. An, W. S. Kim, Y. S. Park, J. M. Moon, D. J. Bae, S. C. Lim, Y. S. Lee and Y. H. Lee, Adv. Funct. Mater., 2001, 11, 387-392.

10 K. H. An, W. S. Kim, Y. S. Park, Y. C. Choi, S. M. Lee, D. C. Chung, D. J. Bae, S. C. Lim and Y. H. Lee, Adv. Mater., 2001, 13, 497-500.

11 J. Chen, A. I. Minett, Y. Liu, C. Lynam, P. Sherrell, C. Wang and G. G. Wallace, Adv. Mater., 2008, 20, 566-570.

12 J. H. Chen, W. Z. Li, D. Z. Wang, S. X. Yang, J. G. Wen and Z. F. Ren, Carbon, 2002, 40, 1193-1197.

13 J. N. Barisci, G. G. Wallace and R. H. Baughman, J. Electrochem. Soc., 2000, 147, 4580-4583.
14 J. N. Barisci, G. G. Wallace, D. Chattopadhyay, F. Papadimitrakopoulos and R. H. Baughman, J. Electrochem. Soc., 2003, 150, E409-E415.

15 A. B. Dalton, S. Collins, E. Munoz, J. M. Razal, V. H. Ebron, J. P. Ferraris, J. N. Coleman, B. G. Kim and R. H. Baughman, Nature, 2003, 423, 703-703.

16 S. Talapatra, S. Kar, S. K. Pal, R. Vajtai, L. Ci, P. Victor, M. M. Shaijumon, S. Kaur, O. Nalamasu and P. M. Ajayan, Nat. Nanotechnol., 2006, 1, 112-116.

17 R. H. Baughman, A. A. Zakhidov and W. A. de Heer, Science, 2002, 297, 787-792.

18 K. P. Gong, F. Du, Z. H. Xia, M. Durstock and L. M. Dai, Science, $2009,323,760-764$.

19 A. Izadi-Najafabadi, S. Yasuda, K. Kobashi, T. Yamada, D. N. Futaba, H. Hatori, M. Yumura, S. Iijima and K. Hat, $A d v$. Mater., 2010, 22, E235-E241.

20 F. Pico, J. M. Rojo, M. L. Sanjuan, A. Anson, A. M. Benito, M. A. Callejas, W. K. Maser and M. T. Martinez, J. Electrochem. Soc., 2004, 151, A831-A837.

21 C. Y. Liu, A. J. Bard, F. Wudl, I. Weitz and J. R. Heath, Electrochem. Solid-State Lett., 1999, 2, 577-578.

22 P. W. Ruch, R. Kotz and A. Wokaun, Electrochim. Acta, 2009, 54, 4451-4458.

23 M. Kaempgen, C. K. Chan, J. Ma, Y. Cui and G. Gruner, Nano Lett., 2009, 9, 1872-1876.

24 X. Zhao, B. T. T. Chu, B. Ballesteros, W. L. Wang, C. Johnston, J. M. Sykes and P. S. Grant, Nanotechnology, 2009, 20, 065605.

25 D. N. Futaba, K. Hata, T. Yamada, T. Hiraoka, Y. Hayamizu, Y. Kakudate, O. Tanaike, H. Hatori, M. Yumura and S. Iijima, Nat. Mater., 2006, 5, 987-994.

26 W. J. Ma, L. Song, R. Yang, T. H. Zhang, Y. C. Zhao, L. F. Sun, Y. Ren, D. F. Liu, L. F. Liu, J. Shen, Z. X. Zhang, Y. J. Xiang, W. Y. Zhou and S. S. Xie, Nano Lett., 2007, 7, 2307-2311.

27 P. M. Ajayan, L. S. Schadler, C. Giannaris and A. Rubio, $A d v$. Mater., 2000, 12, 750-753.

28 S. Toth, M. Fule, M. Veres, J. R. Selman, D. Arcon, I. Pocsik and M. Koos, Thin Solid Films, 2005, 482, 207-210.

29 X. B. Zhang, K. L. Jiang, C. Teng, P. Liu, L. Zhang, J. Kong, T. H. Zhang, Q. Q. Li and S. S. Fan, Adv. Mater., 2006, 18, 15051510

30 B. Xu, F. Wu, S. Chen, C. Z. Zhang, G. P. Cao and Y. S. Yang, Electrochim. Acta, 2007, 52, 4595-4598.

31 R. Shah, X. F. Zhang and S. Talapatra, Nanotechnology, 2009, 20, 395202.

32 C. T. Hsieh, Y. W. Chou and W. Y. Chen, J. Solid State Chem., 2008, 12, 663-669.

33 P. C. Chen, G. Shen, S. Sukcharoenchoke and C. Zhou, Appl. Phys. Lett., 2009, 94, 233104.

34 V. L. Pushparaj, M. M. Shaijumon, A. Kumar, S. Murugesan, L. Ci, R. Vajtai, R. J. Linhardt, O. Nalamasu and P. M. Ajayan, Proc. Natl. Acad. Sci. U. S. A., 2007, 104, 13574-13577.

35 C. S. Du and N. Pan, J. Power Sources, 2006, 160, 1487-1494.

36 E. Frackowiak, K. Jurewicz, S. Delpeux and F. Beguin, J. Power Sources, 2001, 97-98, 822-825.

37 E. Frackowiak, Phys. Chem. Chem. Phys., 2007, 9, 1774-1785.

38 C. Portet, P. L. Taberna, P. Simon, E. Flahaut and C. LabertyRobert, Electrochim. Acta, 2005, 50, 4174-4180.

39 S. B. Yang, J. P. Huo, H. H. Song and X. H. Chen, Electrochim. Acta, 2008, 53, 2238-2240. 